\title{
A SAMPLING THEOREM FOR A CLASS OF PSEUDOANALYTIC FUNCTIONS
}

\author{
J. L. SCHIFF AND W. J. WALKER
}

(Communicated by J. Marshall Ash)

\begin{abstract}
The $\mu$-regular class of pseudoanalytic functions satisfy the CauchyRiemann equations for $\Delta u=\mu^{2} u$. A sampling algorithm is given which expresses the Fourier coefficients of these functions as a countable sum of sample values taken around a circle. This representation is obtained using Möbius inversion.
\end{abstract}

\section{INTRODUCTION}

The potential of the strong nuclear force can be described by the solution $e^{-\mu r} / r$ of the elliptic equation

$$
\Delta u=\mu^{2} u \quad(\mu>0) .
$$

This description was proposed by the Japanese physicist Hideki Yukawa, and equation (1) now bears his name. Subsequently, Duffin [2] undertook the development of a Yukawan potential theory, which forms the basis of our present work. As $\mu \rightarrow 0$, a solution of the Yukawa equation becomes a solution of the Laplace equation.

In this article, attention is restricted to the two-dimensional case of (1). Functions which are $C^{2}$-solutions of (1) in a domain of the complex plane are termed panharmonic. They give rise to pseudoanalytic functions $f=u+i v$, where $u$ and $v$ are panharmonic and satisfy the Cauchy-Riemann equations for (1). These pseudoanalytic functions are termed $\mu$-regular, and have an elegant Fourier series representation, the coefficients of which seem to satisfy a new Bieberbach condition (cf. [5]).

Our aim is to give a sampling theorem. In our case it yields an exact representation of the Fourier coefficients of a $\mu$-regular function $f$ by taking a countable set of values of $f$ on the boundary of a circle. The authors in [3] have already given a similar algorithm for the Taylor coefficients of an analytic

Received by the editors October 25, 1988 and, in revised form, February 12, 1990.

1980 Mathematics Subject Classification (1985 Revision). Primary 30G20; Secondary 30B99, $30 \mathrm{E} 10$.

Key words and phrases. Möbius function, panharmonic, $\mu$-regular, pseudoanalytic, sampling theorem. 
function, but a different approach is required in the present case. Here we employ the representation of Fourier cosine coefficients developed by Bruns [1] and Wintner [6] (cf. [4]).

\section{Definitions}

We first give some preliminaries concerning panharmonic and $\mu$-regular functions. The reader is referred to Duffin [2] for details.

Given a function $f(z)=u(x, y)+i v(x, y)$, where $u, v \in C^{2}$ and are real-valued, satisfying the Cauchy-Riemann equations

$$
\begin{aligned}
& \frac{\partial u}{\partial x}=\frac{\partial v}{\partial y}+\mu u, \\
& \frac{\partial u}{\partial y}=-\frac{\partial v}{\partial x}-\mu v,
\end{aligned}
$$

$f(z)$ is called $\mu$-regular. Note that $f(z)$ is pseudoanalytic and that both $u$ and $v$ are panharmonic. We say that $f(z)$ is $\mu$-regular in the closed unit disk $\bar{U}:|z| \leq 1$ if it is $\mu$-regular in an open disk containing $\bar{U}$. As $\mu \rightarrow 0$, a $\mu$-regular function becomes a classical analytic function.

We now have the following Fourier series representation, deduced from Theorems 10 and 21 of [2]:

Theorem 1. Let $f(z)$ be $\mu$-regular in $\bar{U}$. Then, for $|z|=r \leq 1$,

$$
f(z)=\sum_{n=-\infty}^{\infty} c_{n} I_{|n|}(\mu r) e^{i n \theta},
$$

where

$$
c_{n}=\frac{1}{2 \pi I_{n}(\mu)} \int_{0}^{2 \pi} f\left(e^{i \theta}\right) e^{-i n \theta} d \theta, \quad n=0,1,2, \ldots
$$

and $I_{n}$ is the modified Bessel function of the first kind given by

$$
I_{n}(x)=\frac{1}{n !}\left(\frac{x}{2}\right)^{n}\left[1+\frac{(x / 2)^{2}}{1 \cdot(n+1)}+\frac{(x / 2)^{4}}{1 \cdot 2 \cdot(n+1)(n+2)}+\cdots\right] \text {. }
$$

Moreover, the Fourier coefficients of negative index are given by $c_{-n}=\overline{c_{n-1}}$.

The condition $c_{-n}=\overline{c_{n-1}}$ characterizes a $\mu$-regular function and will be significant in the development of the sampling formula obtained in $\S 3$. Also, for $n=0$ and $0<a \leq 1$, we have the mean value property (cf. [2, Theorem 6])

$$
f(0)=\frac{1}{2 \pi I_{0}(\mu a)} \int_{0}^{2 \pi} f\left(a e^{i \theta}\right) d \theta
$$

\section{A SAMPLING FORMULA}

In this section we discuss a sampling formula for Fourier cosine coefficients which has its origins in the work of Bruns [1] and was subsequently developed by Wintner [6] and, more recently, by us [3,4]. 
A key ingredient of the sampling algorithm is the well-known Möbius function, here denoted by $\nu$ instead of $\mu$, from number theory, defined on the positive integers by:

(i) $\nu(1)=1$;

(ii) $\nu(j)=0$ if there is a prime $p$ such that $p^{2} \mid j$;

(iii) if $j=p_{1} p_{2} \cdots p_{\ell}$ is the prime factorization of $j$, and the $p_{i}$ 's are all distinct, then $\nu(j)=(-1)^{\ell}$.

The role which the Möbius function plays in Fourier analysis results from the fact that if $g(x)$ is a function of period $2 \pi$ with $\int_{0}^{2 \pi} g(x) d x=0$, then, formally,

$$
a_{n}=\sum_{k=1}^{\infty} \frac{\nu(k)}{k n} \sum_{m=1}^{k n} g(2 \pi m / k n), \quad n=1,2,3, \ldots
$$

gives the cosine coefficients of $g(x)$. Wintner gave sufficient conditions for the validity of this identity (cf. [6]). The following adaptation of the theorem of Wintner is now used:

Theorem 2. Let $\varphi$ be real-valued of period $2 \pi$ on $|z|=1$, and let $\omega_{k n}=$ $e^{2 \pi i / k n}$. If $\varphi$ has the normalization $\int_{0}^{2 \pi} \varphi\left(e^{i \theta}\right) d \theta=0$ and $\varphi^{\prime} \in \operatorname{Lip}_{1}([0,2 \pi])$, then the Fourier cosine coefficients of $\varphi$ satisfy

$$
a_{n}=\sum_{k=1}^{\infty} \frac{\nu(k)}{k n} \sum_{m=1}^{k n} \phi\left(\omega_{k n}^{m}\right) .
$$

The sampling theorem for $\mu$-regular functions can now be established.

Theorem 3. Let $f(z)$ be $\mu$-regular in $\bar{U}$. Then the coefficients of the Fourier series representation (2) are given by the recursive relation

$$
c_{n}=\frac{1}{I_{n}(\mu)} \sum_{k=1}^{\infty} \frac{\nu(k)}{k n} \sum_{m=1}^{k n}\left[f\left(\omega_{k n}^{m}\right)-f(0) I_{0}(\mu)\right]-\overline{c_{n-1}}, \quad n=1,2,3, \ldots
$$

Proof. Letting $c_{n}=a_{n}+i b_{n}$ and $|z|=r=1$, (2) yields

$$
\begin{aligned}
f(z)= & \sum_{n=-\infty}^{\infty}\left(a_{n} I_{|n|}(\mu) \cos n \theta-b_{n} I_{|n|}(\mu) \sin n \theta\right) \\
& +i \sum_{n=-\infty}^{\infty}\left(b_{n} I_{|n|}(\mu) \cos n \theta+a_{n} I_{|n|}(\mu) \sin n \theta\right)=u(\theta)+i v(\theta) .
\end{aligned}
$$

The cosine terms of $u$ are

$$
\sum_{n=0}^{\infty} a_{n} I_{n}(\mu) \cos n \theta+\sum_{n=1}^{\infty} a_{-n} I_{n}(\mu) \cos n \theta=a_{0} I_{0}(\mu)+\sum_{n=1}^{\infty}\left(a_{n}+a_{-n}\right) I_{n}(\mu) \cos n \theta .
$$

Likewise, the cosine terms of $v$ are:

$$
b_{0} I_{0}(\mu)+\sum_{n=1}^{\infty}\left(b_{n}+b_{-n}\right) I_{n}(\mu) \cos n \theta .
$$


Now, the hypotheses imply that functions $U(\theta)=u(\theta)-a_{0} I_{0}(\mu)$ and $V(\theta)=$ $v(\theta)-b_{0} I_{0}(\mu)$ belong to $\operatorname{Lip}_{1}([0,2 \pi])$. Moreover,

$$
\frac{1}{2 \pi} \int_{0}^{2 \pi} U\left(e^{i \theta}\right) d \theta=\frac{1}{2 \pi} \int_{0}^{2 \pi} u\left(e^{i \theta}\right) d \theta-a_{0} I_{0}(\mu)=0=\frac{1}{2 \pi} \int_{0}^{2 \pi} V\left(e^{i \theta}\right) d \theta .
$$

As a consequence, Theorem 2 implies

$$
\begin{aligned}
& \left(a_{n}+a_{-n}\right) I_{n}(\mu)=\sum_{k=1}^{\infty} \frac{\nu(k)}{k n} \sum_{m=1}^{k n} U\left(\omega_{k n}^{m}\right), \\
& \left(b_{n}+b_{-n}\right) I_{n}(\mu)=\sum_{k=1}^{\infty} \frac{\nu(k)}{k n} \sum_{m=1}^{k n} V\left(\omega_{k n}^{m}\right) .
\end{aligned}
$$

Combining $(4)+i(5)$, we obtain

$$
\left(c_{n}+c_{-n}\right) I_{n}(\mu)=\left(c_{n}+\overline{c_{n-1}}\right) I_{n}(\mu)
$$

by the $\mu$-regularity of $f(z)$, and it follows that

$$
c_{n}=\frac{1}{I_{n}(\mu)} \sum_{k=1}^{\infty} \frac{\nu(k)}{k n} \sum_{m=1}^{k n}\left[f\left(\omega_{k n}^{m}\right)-f(0) I_{0}(\mu)\right]-\overline{c_{n-1}}, \quad n=1,2,3, \ldots
$$

as desired.

As $f(0) I_{0}(\mu)$ is the integral mean of $f$ by (3), this term can be evaluated by various techniques if it is not known explicitly.

\section{TRUNCATION ERRORS}

An estimate can be given for the truncation error for the preceding representation. To this end, we require the following (cf. [6]):

Lemma. If $\varphi$ is a real-valued function of period $2 \pi$ on $|z|=1$ and satisfies $\varphi^{\prime} \in \operatorname{Lip}_{1}([0,2 \pi])$, then

$$
\mid \frac{1}{2 \pi} \int_{0}^{2 \pi} \phi\left(e^{i \theta}\right) d \theta-\frac{1}{n} \sum_{m=1}^{n} \phi\left(e^{2 \pi i m / n)} \mid \leq \frac{C}{n^{2}},\right.
$$

where $C$ is the Lipschitz constant.

Assuming no error in $c_{n-1}$, the following estimate obtains, using a truncated sum in Theorem 3:

$$
\begin{aligned}
\left|c_{n}-\left(\frac{1}{I_{n}(\mu)} \sum_{k=1}^{N} \frac{\nu(k)}{k n} \sum_{m=1}^{k n} f\left(\omega_{k n}^{m}\right)-\overline{c_{n-1}}\right)\right| & =\left|\left(\frac{1}{I_{n}(\mu)} \sum_{k=N+1}^{\infty} \frac{\nu(k)}{k n} \sum_{m=1}^{k n} f\left(\omega_{k n}^{m}\right)\right)\right| \\
& \leq \frac{1}{I_{n}(\mu)} \cdot \frac{2 C}{n^{2}} \sum_{k=N+1}^{\infty} \frac{1}{k^{2}} \\
& \leq \frac{2 C}{I_{n}(\mu) n^{2} N},
\end{aligned}
$$


where we have taken the normalization $f(0)=0$, and applied the lemma to the real and imaginary parts of $f(z)$.

Note that, although the error in computing $c_{n}$ is cumulative, this is somewhat compensated for by the $n^{-2}$ factor in the estimate. In fact, computer implementation of the sampling algorithm would seem to indicate much more rapid convergence than is given by the error estimate.

\section{REFERENCES}

1. H. Bruns, Grundlinier des Wissenschaftlichen Rechrens, Leipzig, 1903.

2. R. J. Duffin, Yukawan potential theory, J. Math. Anal. Appl. 35 (1971), 104-130.

3. J. L Schiff and W. J. Walker, A sampling theorem for analytic functions, Proc. Amer. Math. Soc. 99 (1987), 737-740.

4. _ A sampling theorem and Wintner's results on Fourier coefficients, J. Math. Anal. Appl. 133 (1988), 466-471.

5. __ A Bieberbach condition for a class of pseudo-analytic functions, J. Math. Anal. Appl. 146 (1990), 570-579.

6. A Wintner, An arithmetical approach to ordinary Fourier series, Waverly Press, 1945.

Department of Mathematics and Statistics, University of Auckland, Auckland, New ZEALAND 